Pacific Journal of Mathematics

AN EHRENFEUCHT GAME FOR THE MULTIVARIABLE
QUANTIFIERS OF MALITZ AND SOME APPLICATIONS 


\title{
AN EHRENFEUCHT GAME FOR THE MULTIVARIABLE QUANTIFIERS OF MALITZ AND SOME APPLICATIONS
}

LEE BADGER

\begin{abstract}
An Ehrenfeucht game is developed for some languages which admit the Malitz quantifiers. The method is used to show that equivalence of structures with respect to such languages is usually preserved by the cardinal sum operation, and that such equivalence need not be preserved by the direct product operation. Craig's theorem is shown to fail in all cardinal interpretations of the languages.
\end{abstract}

In this paper we report the results of a study of the model theory of $L_{\kappa}^{<\omega} \cdot L_{\kappa}^{<\omega}$ is essentially the union of the languages $L_{\kappa}^{n}$ where $L^{0}$ is the first order predicate calculus and for $n>0 L_{\kappa}^{n}$ is an extension of $L^{0}$ obtained by allowing the additional quantifier symbol $Q^{n}$ which binds $n$ distinct variables and $Q^{n} x_{1}, \cdots, x_{n} \varphi$ has the interpretation: "there is a set of individuals $X$ of power $\kappa$ such that for distinct $x_{1}, \cdots, x_{n} \in X, \varphi . "$ In this study the principal tool is an Ehrenfeucht game and the main results are that $L^{<\omega}$ equivalence is preserved by cardinal sums of structures, that $L^{<\omega}$ equivalence need not be preserved by direct products of structures, and that Craig's theorem fails in $L^{<\omega}$.

Using our notation $L^{1}$ is the language $L(Q)$ studied by Keisler [12], Fuhrken [9], [10], Vaught [17] and others. $L^{<\omega}$ is an extension of $L^{1}$ which was introduced by Magidor and Malitz in [14]. In this paper they show that with $L_{\omega_{1}}^{<\omega}$ one can say without the use of additional symbols that an equivalence relation has uncountably many classes, that a tree order is a Suslin tree, and that a group has an uncountable abelian subgroup. To show that this is significantly more expressiveness than is possible with $L_{\omega_{1}}^{1}$ they point out models of set theory where the notion of a Suslin tree cannot be characterized in $L_{\omega_{1}}^{1}$ even with the use of relativized reducts. They show that under certain set theoretical assumptions $L_{\omega_{1}}^{<\omega}$ is countably compact.

1. Preliminaries. The class of infinite cardinals is denoted by card. $\kappa$ and $\lambda$ usually denote infinite cardinals. $c X$ denotes the cardinality of $X$ and ef $\kappa$ denotes the cofinality of $\kappa$.

$L^{0}$ denotes the ordinary first order predicate calculus with its customary rules for formations of formulas. If $n \in \omega-1, L^{n}$ denotes the language which extends $L^{0}$ by admitting the additional quantifier symbol $Q^{n}$ and with the additional rule of formation that says if $\phi$ 
is a formula of $L^{n}$ and $x_{1}, \cdots, x_{n}$ are distinct variables then $Q^{n} x_{1}, \cdots$, $x_{n} \varphi$ is also a formula of $L^{n}$. $L^{<\omega}$ is the extension of $L^{0}$ which admits all the quantifier symbols $Q^{n}$. If $\mathfrak{A}$ is a structure and $\kappa$ an infinite cardinal we write $\mathfrak{U} \vDash{ }_{k} Q^{n} x_{1}, \cdots, x_{n} \varphi$ if there is $X \subseteq \mathfrak{A}$ such that $c X \geqq \kappa$ and for distinct $a_{1}, \cdots, a_{n} \in X, \mathfrak{A} \models_{\kappa} \phi\left[a_{1}, \cdots, a_{n}\right] . \quad \kappa$ is called the cardinal interpretation of $Q$. If $\kappa$ is fixed throughout a discussion or is clear from the context we may omit the subscript $\kappa$ and simply write $\vDash$. Also if we wish to discuss the languages $L^{n}$ or $L^{<\omega}$ with the interpretation, $\kappa$, fixed throughout we refer to the languages as $L_{\kappa}^{n}$ or $L_{\kappa}^{<\omega}$.

The language $\underline{L}$ is an extension of $L^{0}$ which admits the additional quantifier symbols $\bar{Q}_{\lambda}^{n}$ for $n \in \omega-1$ and $\lambda \in$ card; and admits conjunctions and disjunctions of any cardinal length. Satisfaction for $\underline{L}$ is defined as expected when $Q_{\lambda}^{n}$ is treated like $Q^{n}$ with the $\lambda$-interpretation.

It will be convenient to assume our languages have quantifiers dual to the $Q^{n}$. We denote these by $\delta^{n}$ and define satisfaction of them by $\mathfrak{A} \vDash{ }_{\kappa} \mathcal{O}^{n} x_{1}, \cdots, x_{n} \varphi$ if and only if $\mathfrak{A} \vDash_{\kappa} \neg Q^{n} x_{1}, \cdots, x_{n}-\varphi$. If $L$ is a language, two structures are said to be $L$ equivalent if they satisfy exactly the same sentences of $L$; we denote this by $\mathfrak{A} \equiv \mathfrak{B}(L)$. For a precise definition of the elementary terms not defined here we refer the reader to [4].

2. Ehrenfeucht games. Classical Ehrenfeucht games provide a test for determining $L^{0}$ equivalence of structures. The game theoretic form of this method was first developed by Ehrenfeucht [6] but a similar method was known to Fraissé [8] in the form of $n$-equivalence between structures. Lipper [13], Brown [3], and Vinner [18] independently extended the method to $L^{1}$. In this section we extend the games to $L^{n}, L^{<\omega}$, and $\underline{L}$.

Suppose $\mathfrak{A}$ and $\mathfrak{B}$ are similar structures (i.e., of the same type), $m$ and $n$ are positive integers, and $\kappa$ is an infinite cardinal. We describe $G_{m}^{n}(\mathfrak{A}, \mathfrak{B})_{\kappa}$, a game played by two players denoted I and II. $G_{m}^{n}(\mathfrak{A}, \mathfrak{B})_{\kappa}$ consists of $m$ periods of play. During each period of play $n$ or less elements are chosen from $|\mathfrak{A}|$ and the same number of elements are chosen from $|\mathfrak{B}|$. We describe a typical period of play, the $j$ th period, with all others being similar.

Player I begins by choosing a structure, say $\mathfrak{A}$. If I chooses $\mathfrak{B}$ then the roles of $\mathscr{A}$ and $\mathfrak{B}$ are reversed in the following description. Player I may make either of two types of moves.

Type 1. Player I chooses a sequence of $n$ or less elements from $|\mathfrak{A}|, a_{j_{1}}, \cdots, a_{j k_{j}} \cdot$ 
Player II then responds with a sequence of $k_{j}$ elements from $|\mathfrak{B}|$, $b_{j 1}, \cdots, b_{j k_{j}}$.

Type 2. Player I chooses a set $X \subseteq|\mathfrak{A}|$ with $c X \geqq \kappa$.

Plays II responds with $Y \subseteq|\mathfrak{B}|$ with $c Y \geqq \kappa$.

Player I chooses a sequence of $n$ or less distinct elements from $Y, b_{j 1}, \cdots, b_{j k_{j}}$.

Player II responds with a sequence of $k_{j}$ distinct elements from $X, a_{j 1}, \cdots, a_{j_{k j}}$.

This completes the description of period $j$. The completion of period $m$ constitutes the completion of $G_{m}^{n}(\mathfrak{A}, \mathfrak{B})_{\kappa}$. Many times we omit the subscript $\kappa$ when it is clear from the context what $\kappa$ is, in particular, when the language under discussion is $L_{\kappa}^{<\omega}$ or $L_{\kappa}^{n}$. In the future we may refer to a move of Type 1 as an "existential move" and a move of Type 2 as a " $Q$ move."

Let $O_{l}=\left\{\left(a_{j i}, b_{j i}\right): 1 \leqq j \leqq l\right.$ and $\left.1 \leqq i \leqq k_{j}\right\}$ for $l \leqq m$ and let $O=O_{m} . \quad O$ is called the outcome of $G_{m}^{n}(\mathfrak{A}, \mathfrak{B})_{\kappa}$ and $O_{l}$ for $l<m$ is called a partial outcome. We say that player II wins $G_{m}^{n}(\mathfrak{A}, \mathfrak{B})_{\kappa}$ if $O$ is an isomorphism from its domain to its range when each is viewed as a substructure of $\mathfrak{A}$ and $\mathfrak{B}$ respectively. We say that player I wins if player II does not win. By a winning strategy for player II in $G_{m}^{n}(\mathfrak{A}, \mathfrak{B})_{\kappa}$ we mean a fixed procedure that player II can follow so that no matter how player I moves, player II wins. If $O_{l}$ is a partial outcome of $G_{m}^{n}(\mathfrak{A}, \mathfrak{B})_{\kappa}$ we say that player II is in a winning position or that $O_{l}$ is a winning position for player II if there is a fixed procedure that player II can follows to finish $G_{m}^{n}(\mathfrak{A}, \mathfrak{B})_{\kappa}$ such that no matter how player I moves, player II wins.

THEOREM 2.1. Suppose $\mathfrak{A}$ and $\mathfrak{B}$ are of the same finite type and $n$ is a positive integer. Then $\mathfrak{A} \equiv \mathfrak{B}\left(L_{\kappa}^{n}\right)$ if and only if for each positive integer $m$ player II has a winning strategy in $G_{m}^{n}(\mathfrak{A}, \mathfrak{B})_{\kappa}$.

We omit the proof because the method parallels closely that used for $L_{\kappa}^{1}$ and that method appears in the literature in [18]. A detailed proof of Theorem 2.1 appears in [2].

Corollary 2.2. Suppose $\mathfrak{A}$ and $\mathfrak{B}$ are of the same finite type. Then $\mathfrak{A} \equiv \mathfrak{B}\left(L_{\kappa}^{<\omega}\right)$ if and only if for each pair of positive integers $m$ and $n$, player II has a winning strategy in $G_{m}^{n}(\mathfrak{A}, \mathfrak{B})_{\kappa}$.

This follows from Theorem 2.1 by noting that for each $\varphi \in L^{<\omega}$ there is an $n \in \omega-1$ and $\psi \in L^{n}$ such that $\vDash_{\kappa} \varphi \leftrightarrow \psi$.

In practice when using the games to show two structures fully 
equivalent we may assume that when player I makes an existential move he chooses exactly one element. This is sufficient to imply the structures equivalent, for if player I could win $G_{m}^{n}(\mathfrak{A}, \mathfrak{B})_{\kappa}$ by choosing $\leqq n$ elements on each existential move, then there would be a $k \leqq$ $m \cdot n$ such that player I could win $G_{k}^{n}(\mathfrak{A}, \mathfrak{B})_{\kappa}$ by choosing only one element in each existential move.

We now define a game for determining $\underline{L}$ equivalence of structures. This game we denoted by $G(\mathfrak{A}, \mathfrak{B})$. It is like $G_{m}^{n}(\mathfrak{A}, \mathfrak{B})_{\kappa}$ except for the following differences:

(i) there are $\omega$ periods of play,

(ii) there is no bound on the length of the finite sequence of elements that player I chooses in each period,

(iii) in a move of type two player I may choose any infinite subset of a structure, and player II must respond with a subset of the other structure of equal or greater cardinality than the cardinality of that subset chosen by player I.

The definitions of outcome, partial outcome, winning, and winning strategy for $G(\mathfrak{A}, \mathfrak{B})$ are analogous to those definitions for $G_{m}^{n}(\mathfrak{A}, \mathfrak{B})_{\kappa}$.

THEOREM 2.3. Supose $\mathfrak{A}$ and $\mathfrak{B}$ are of the same type. Then $\mathfrak{A} \equiv \mathfrak{B}(\underline{L})$ if and only if player II has a winning strategy in $G(\mathfrak{A}, \mathfrak{B})$.

Again we omit the proof. A proof does appear in [2], and it makes use of methods similar to those of Karp in [11].

\section{Cardinal sums and $L^{<\omega}$ equivalence.}

Definition 3.1. Spupose $\mathfrak{A}_{i}(i \in I)$ are similar relational structures. We define in the usual way the cardinal sum of $\mathfrak{A}_{i}(i \in I)$, denoted $\sum_{i \in I} \mathfrak{A}_{i}$. First, assume that $\left|\mathfrak{A}_{i}\right| \cap\left|\mathfrak{A}_{j}\right|=0$ for $i \neq j$. If this is not the case, replace certain $\mathfrak{A}_{i}$ by suitable isomorphic copies. Suppose $\sum_{i \in I} \mathfrak{A}_{i}$ is denoted by $\mathfrak{A}$. Then $|\mathfrak{A}|=\bigcup_{i \in I}\left|\mathfrak{A}_{i}\right|$ and if $R$ is in the common type, $R^{\mathfrak{\imath}}=\bigcup_{i \in I} R^{\Re_{i}}$.

THEOREM 3.2. Suppose $\kappa$ is regular and $\mathfrak{A}_{i} \equiv \mathfrak{B}_{i}\left(L_{k}^{<\omega}\right)$ for all $i \in I$. Then $\sum_{i \in I} \mathfrak{A}_{i} \equiv \sum_{i \in I} \mathfrak{B}_{i}\left(L_{\kappa}^{<\omega}\right)$.

Proof. Let $\mathfrak{A}=\sum_{i \in I} \mathfrak{A}_{i}$ and $\mathfrak{B}=\sum_{i \in I} \mathfrak{B}_{i}$. Since each sentence involves only finitely many relation symbols, it suffices to show that $\left.\left.\mathfrak{A}\right|_{\tau} \equiv \mathfrak{B}\right|_{\tau}\left(L_{\kappa}^{<\omega}\right)$ for each finite type $\tau$. So we may assume that the type of the structures is finite. Let $m, n \in \omega-1$ be given, we will show that player II has a winning strategy in $G_{m}^{n}(\mathfrak{A}, \mathfrak{B})$. Explicitly, we will show that player II can play $G_{m}^{n}(\mathfrak{A}, \mathfrak{B})$ such that for each $(a, b)$ in the outcome we have $a \in\left|\mathfrak{A}_{i}\right|$ if and only if 
$b \in\left|\mathfrak{B}_{i}\right|$ and such that if $O_{i}=O \cap\left|\mathfrak{A}_{i}\right| \times\left|\mathfrak{B}_{i}\right|$ then $O_{i}$ is a winning position for player II in $G_{m}^{n}\left(\mathfrak{H}_{i}, \mathfrak{B}_{i}\right)$. These two facts mean that player II has a win, for if $\varphi\left(v_{1}, \cdots, v_{n}\right)$ is an atomic formula and $a_{1}, \cdots, a_{n}$ do not all come from a single $\left|\mathfrak{A}_{i}\right|$, then $\mathfrak{A} \not \neq \varphi\left[a_{1}, \cdots, a_{n}\right]$; and similarly $\mathfrak{B} \not \neq \varphi\left[b_{1}, \cdots b_{n}\right]$ when not all of $b_{1}, \cdots, b_{n}$ come from a single $\left|\mathfrak{B}_{i}\right|$.

We suppose inductively that player II can play so that through $j-1$ periods the above holds, where for $O$ we mean that portion of the outcome constructed in the first $j-1$ periods.

Case 1. Suppose player I chooses $a_{j} \in\left|\mathfrak{A}_{i}\right| \subseteq|\mathfrak{A}|$. Player II considers $a_{j}$ as a choice made by player $I$ in $G_{m}^{n}\left(\mathfrak{H}_{i}, \mathfrak{B}_{i}\right)$. Player II has a strategy in this game, since $\mathfrak{A}_{i} \equiv \mathfrak{B}_{i}\left(L_{\kappa}^{<\omega}\right)$ and since the type is finite. Also, by induction, player II is in a winning position in this game. $G_{m}^{n}\left(\mathfrak{A}_{i}, \mathfrak{B}_{i}\right)$ may or may not be in period $j$, depending upon how many elements have been chosen from the $i$ th summand thus far. But this is not a factor in the argument. Certainly the play in $G_{m}^{n}\left(\mathfrak{A}_{i}, \mathfrak{B}_{i}\right)$ is not beyond the $j$ th period. So the strategy in $G_{m}^{n}\left(\mathfrak{A}_{i}, \mathfrak{B}_{i}\right)$ gives $b \in\left|\mathfrak{B}_{i}\right|$, which player II chooses as a $b \in|\mathfrak{B}|$. The induction assumption is satisfied.

Case 2. Suppose player I chooses $X \subseteq|\mathfrak{A}|, c X \geqq \kappa$.

Subcase a. Suppose there is some $i \in I$ such that $c\left(X \cap\left|\mathfrak{A}_{i}\right|\right) \geqq \kappa$. Then player II considers $X \cap\left|\mathfrak{A}_{i}\right|$ as a choice made by player I in $G_{m}^{n}\left(\mathfrak{A}_{i}, \mathfrak{B}_{i}\right)$ and the reasoning is the same as in case 1.

Subcase b. Suppose there is no $i \in I$ such that $c\left(X \cap\left|\mathfrak{A}_{i}\right|\right) \geqq \kappa$. Then since $\kappa$ is regular, $c M \geqq \kappa$ where $M=\left\{i \in I: X \cap\left|\mathfrak{A}_{i}\right| \neq 0\right\}$. For each $i \in M$, pick a single $x_{i} \in X \cap\left|\mathfrak{A}_{i}\right|$. Player II considers $x_{i}$ as a choice made by player $I$ in a move of Type $I$ in $G_{m}^{n}\left(\mathfrak{A}_{i}, \mathfrak{B}_{i}\right)$, for each $i \in M$. The strategies in each of these games give $y_{i} \in\left|\mathfrak{B}_{i}\right|$ for $i \in M$. For $Y \subseteq|\mathfrak{A}|$, player II chooses $Y=\left\{y_{i}: i \in M\right\} . \quad c Y \geqq \kappa$ since $c M \geqq \kappa$. No matter what distinct $b_{j 1}, \cdots, b_{j n} \in Y$ that player I chooses, there are $n$ distinct indices $i_{1}, \cdots, i_{n} \in M$ such that $b_{j k}=y_{j_{k}}, k=1, \cdots, n$. For $a_{j 1}, \cdots, a_{j_{n}} \in X$, player II chooses $a_{j k}=x_{i_{k}}, k=1, \cdots, n$. Again the induction hypothesis is satisfied.

REMARK. If $\kappa$ is singular the theorem still holds if $c I<\operatorname{cf} \kappa$. The proof is the same, except Subcase $b$ of Case 2 will not arise, and this is the only place where the regularity of $\kappa$ was used.

If $\lambda=$ cf $\kappa<\kappa$ and $\mu=c I \geqq \lambda$ we have this counterexample. Suppose $\kappa=\sum_{i \in \lambda} \kappa_{i}$ where $\omega \leqq \kappa_{i}<\kappa$ for all $i \in \lambda$. The type of the structures in our counterexample will have one unary relation, 
$U$. For $i \in \lambda$ set $\mathfrak{A}_{i}=\left\langle\kappa_{i}, \kappa_{i}\right\rangle$ and $\mathfrak{B}_{i}=\langle\omega, \omega\rangle$. For $i \in \mu-\lambda$ set $\mathfrak{B}_{i}=\langle 1,0\rangle$ and $\mathfrak{B}_{i}=\langle 1,0\rangle$. Because of the cardinalities involved it is clear that $\mathfrak{A}_{i} \equiv \mathfrak{B}_{i}\left(L_{\kappa}^{<\omega}\right)$ for all $i \in \mu$. However $c U$ in $\sum_{i \in I} \mathfrak{A}_{i}$ is $\sum_{i \in \lambda} \kappa_{i}=\kappa$ and $c U$ in $\sum_{i \in I} \mathfrak{B}_{i}$ is $\sum_{i \in \lambda} \omega=\omega \cdot \lambda=\lambda<\kappa$. So $\sum_{i \in I} \mathfrak{A}_{i} \vDash$ $Q x U(x)$ whereas $\sum_{i \in I} \mathfrak{B}_{i} \not \neq Q x U(x)$. So $\sum_{i \in I} \mathfrak{A}_{i} \not \equiv \sum_{i \in I} \mathfrak{B}_{i}\left(L^{1}\right)$.

DEFINITION 3.3. Suppose $\lambda$ is a cardinal, $\mathfrak{A}$ a structure. By $\mathfrak{A} \cdot \lambda$ we mean $\sum_{i \in \lambda} \mathfrak{A}_{i}$ where $\mathfrak{A}_{i} \cong \mathfrak{A}$ for each $i \in I$ and $\left|\mathfrak{A}_{i}\right| \cap\left|\mathfrak{A}_{j}\right|=0$ for $i \neq j$.

A natural extension of the question of preservation of $L^{<\omega}$ equivalence by cardinal sums is the following: When is it the case that $\mathfrak{A} \equiv \mathfrak{B}\left(L_{\kappa}^{<\omega}\right)$ and $\lambda \equiv \mu\left(L_{\kappa}^{<\omega}\right)$ imply $\mathfrak{A} \cdot \lambda \equiv \mathfrak{B} \cdot \mu\left(L_{\kappa}^{<\omega}\right)$ ? Of course, $\lambda \equiv$ $\mu\left(L_{\kappa}^{<\omega}\right)$ just means that $\lambda$ and $\mu$ are the same finite cardinal or $\lambda$ and $\mu$ are both infinite and less than $\kappa$ or $\lambda$ and $\mu$ are both greater than or equal to $\kappa$. The answer is given the following theorem and counterexample. We omit the proof. The interested reader is referred to $[2]$.

THEOREM 3.4. Suppose $\mathfrak{A} \equiv \mathfrak{B}\left(L_{\kappa}^{<\omega}\right)$ and $\lambda \equiv \mu\left(L_{\kappa}^{<\omega}\right)$ and suppose it is not the case that cf $\kappa \leqq \lambda+\mu<\kappa$. Then $\mathfrak{A} \cdot \lambda \equiv \mathfrak{B} \cdot \mu\left(L_{\kappa}^{<\omega}\right)$.

Counterexample 3.5. Suppose cf $\kappa \leqq \lambda+\mu<\kappa$. Then there are structures $\mathfrak{A}$ and $\mathfrak{B}$ such that $\mathfrak{A} \equiv \mathfrak{B}\left(L_{\kappa}^{<\omega}\right)$ and $\mathfrak{A} \cdot \lambda \not \equiv \mathfrak{B} \cdot \mu\left(L_{\kappa}^{2}\right)$.

4. Direct products and $L^{<\omega}$ equivalence. It is well known that if $\mathfrak{A}_{i} \equiv \mathfrak{B}_{i}\left(L^{0}\right)$ for $i \in I$ then $\prod_{i \in I} \mathfrak{A}_{i} \equiv \prod_{i \in I} \mathfrak{B}_{i}\left(L^{0}\right)$ (here $\Pi$ denotes the ordinary direct product of structures). This was first shown by Mostowski [15], and was considerably generalized by Feferman and Vaught in [7]. In this work they show that a very general type of product of structures preserves the notion of elementary equivalence between its factors. As special cases they obtained preservation theorems for cardinal sum and direct product, among others. Wojciechowska [19] extended their work to the language $L^{1}$ and obtained preservation theorems with some restrictions placed on the interpretation $\kappa$, and on the size of the index set. Lipner [13] also obtained similar results.

In this section we will see that the situation for $L^{<\omega}$ is quite different. Using the Ehrenfeucht games (which in $L^{0}$ gives an immediate proof of the positive result) we will produce counterexamples which show that $L^{<\omega}$ equivalence need not be preserved under products. In fact we produce structures $\mathfrak{A}$ and $\mathfrak{B}$ such that $\mathfrak{A} \equiv \mathfrak{B}\left(L^{<\omega}\right)$ but $\mathfrak{A}^{2} \not \equiv \mathfrak{B}^{2}\left(L^{2}\right)$. Here $\mathfrak{A}^{2}$ denotes $\mathfrak{A} \times \mathfrak{A}$. 
Definition 4.1. A linear order is said to be $\kappa$-dense if between any two elements there are $\kappa$ elements.

THEOREM 4.2. Suppose $\mathfrak{A}$ and $\mathfrak{B}$ are $\kappa$-dense linear orders without endpoints of power $\kappa$. Then $\mathfrak{A} \equiv \mathfrak{B}(\underline{L})$.

Proof. We will show player II has a winning strategy in $G(\mathfrak{A}, \mathfrak{B})$.

Let $O_{j}$ denote that portion of the outcome selected through the first $j$ periods. Say $O_{j}=\left\{\left(a_{i}, b_{i}\right): i<k_{j}<\omega\right\}$. We will show that player II can play such that for each $j$ there is permutation $\pi_{j}: k_{j} \rightarrow$ $k_{j}$ such that

$$
\begin{aligned}
& a_{\pi_{j}(0)} \leqq a_{\pi_{j}(1)} \leqq \cdots \leqq a_{\pi^{j}\left(k_{j}-1\right)} \\
& b_{\pi_{j}(0)} \leqq b_{\pi_{j}(1)} \leqq \cdots \leqq b_{\pi_{j}\left(k_{j}-1\right)}
\end{aligned}
$$

with equality holding in the first list if and only if it holds at the corresponding place in the second list. The existence of such $\pi_{j}$ for each $j \in \omega$ implies that player II has a win in $G(\mathfrak{A}, \mathfrak{B})$.

We assume by induction, that player II can play so that at the end of period $j\left(^{*}\right)$ holds. Without loss of generality we may assume $\pi_{j}$ is the identity. We describe player II's strategy during period $j+1$.

Case 1. Suppose player I chooses a single element, $a \in|\mathfrak{A}|$. If $a$ equals some previously chosen $a_{i}$, then player II chooses $b$ to be the corresponding $b_{i}$. Otherwise, either $a<a_{0}$ or $a>a_{k_{j-1}}$ or there is $i \in k_{j}$ such that $a_{i}<a<a_{i+1}$. If $a<a_{0}$ then player II chooses $b$ to be any element $<b_{0}$; this can be done because $\mathfrak{B}$ has no endpoints. If $a>a_{k_{j-1}}$, player II chooses $b>b_{k_{j-1}}$. If $a_{i}<a<a_{i+1}$ player II chooses $b$ to be any element in $\mathfrak{B}$ such that $b_{i}<b<b_{i+1}$. This can be done because $\mathfrak{B}$ is a dense linear order. In any of these cases it is clear that there is $\pi_{j+1}: k_{j+1} \rightarrow k_{j+1}$ such that $\left({ }^{*}\right)$ holds.

Case 2. Suppose player I chooses $X \leqq|\mathfrak{A}|$ with $\omega \leqq \lambda=c X \leqq \kappa$. First note that $a_{0}, \cdots, a_{k_{j}-1}$ partition $|\mathfrak{X}|$ into $k_{j}+1$ segment. So there is at least one of these segments, say $\left(a_{i}, a_{i+1}\right)$ such that $\left|X \cap\left(a_{i}, a_{i+1}\right)\right| \geqq \lambda$. Here $\left(a_{i}, a_{i+1}\right)$ denotes $\left\{x: a_{i}<x<a_{i+1}\right\}$. If it happens that the only segment with the large intersection is the initial or terminal segment, the argument is similar. Since $a_{i}<a_{i+1}$, by induction $b_{i}<b_{i+1}$; and since $\mathfrak{B}$ is $\kappa$-dense, there are $\kappa$ elements between $b_{i}$ and $b_{i+1}$. For $Y \subseteq|\mathfrak{B}|$, player II chooses the set of elements strictly between $b_{i}$ and $b_{i+1}$. Since $\mathfrak{B}$ is $\kappa$-dense, this is a set of power $\kappa$. Now suppose player I chooses distinct $b_{(j+1) 1}, \cdots$, $b_{(j+1) n} \in Y$. All of these are strictly between $b_{i}$ and $b_{i+1}$. Since there 
are $\lambda$ elements of $X$ strictly between $a_{i}$ and $a_{i+1}$, it is clear that player II can choose $a_{(j+1)}, \cdots, a_{(j+1) n} \in X$ so that the induction hypothesis is satisfied.

LEMMA 4.4. Suppose $\mathfrak{A}=\left\langle A, \leqq^{\mathfrak{M}}\right\rangle$ is a reflexive linear order. Then the following are equivalent:

(i) $\mathfrak{U}^{2}$ has an antichain of power $\kappa$.

(ii) $\quad \mathfrak{A}^{2} \vDash{ }_{k} Q^{2} x y[\neg(x \leqq y) \wedge \neg(y \leqq x)]$.

(iii) There is $f \subseteq|\mathfrak{A}|^{2}$ so that $f$ is a strictly decreasing function and cardinality of $f$ is $\geqq \kappa$.

Proof. (i) $\Leftrightarrow$ (ii) follows immediately from the definition of antichain and satisfaction. Suppose (ii). Then there is $X \cong\left|\mathfrak{A}^{2}\right|$ so that for distinct $x, y \in X, \neg(x \leqq y)$ and $\neg(y \leqq x) . \quad X$ is our candidate for $f$. Let $x=\left(x_{1}, x_{2}\right)$ and $y=\left(y_{1}, y_{2}\right)$. By definition of $\leqq$ in $\mathfrak{A}^{2}$ we have (since $\neg x \leqq y$ ) that $x_{1}<^{\mathfrak{x}} y_{1}$ implies $x_{2} \varliminf^{\mathfrak{x}} y_{2}$. Since $\leqq^{\mathfrak{x}}$ is a linear order this means that $x_{1} \leqq{ }^{x} y_{1}$ implies $y_{2}<x_{2}$. Also $x_{1} \neq y_{1}$ for if $x_{1}=y_{1}$ then since $x_{2} \leqq y_{2}$ or $y_{2} \leqq x_{2}$ we would have $x \leqq y$ or $y \leqq x$. This establishes (iii). A similar argument shows that (ii) follows from (iii) by showing that $f$ is a satisfying set.

In order to construct the counterexamples we need the existence of a certain type of linear order.

LeMma 4.5. Suppose $\kappa>\omega$. Then there is a reflexive linear order $\mathfrak{A}$ such that $\mathfrak{A}$ is $\kappa$-dense, $\mathfrak{A}$ is of power $\kappa, \mathfrak{A}$ has no end points, $\mathfrak{U}$ has a suborder of type $\kappa$, and $\mathfrak{X}^{2}$ has no antichain of power $\kappa$.

Proof. We begin the construction by constructing a sequence of order types $\mu_{n}, 1 \leqq n<\omega$ such that for each $n, \mu_{n}$ is isomorphic to the ordinal $\kappa^{n}$, and for each $n, \mu_{n} \subseteq \mu_{n+1}$.

Let $\mu_{1}=\kappa$ with the usual order. Given $\mu_{n}, \mu_{n+1}$ results from $\mu_{n}$ by placing a copy of $\kappa$ strictly between $\alpha$ and $\alpha+1$ for each $\alpha \in \mu_{n}$.

It is clear that $\mu_{n} \subseteq \mu_{n+1}$, in fact it is a subordering. Let $\mu=$ $\mathrm{U}_{1 \leqq n<\omega} \mu_{n}$ with the induced order. $\mu$ has the following properties:

(i) $c \mu=\kappa$.

(ii) $\mu$ has a first element and no last element.

(iii) $\mu$ is $\kappa$-dense.

(iv) $\mu$ has a suborder of type $\kappa$.

(v) $\mu^{2}$ has no antichain of power $\kappa$.

Only (v) needs verification. We check condition (iii) of Lemma 4.4. Suppose $X \subseteq \mu, c X=\omega_{1}$ and $f: X \rightarrow \mu$. We claim $f$ cannot be strictly decreasing; this will establish (v). Suppose $f$ is strictly decreasing. 
Let $X_{n}=X \cap \mu_{n}$ for $0<n<\omega . \quad X=\bigcup X_{n}$. Since $c X=\omega_{1}$ there is an $n \in \omega$ such that $c X_{n}=\omega_{1}$, so we have $f: X_{n} \rightarrow \mu$ and $X_{n} \subseteq \mu_{n}$. Let $Y=f\left(X_{n}\right) . \quad c Y=\omega_{1}$ since $f$ is strictly decreasing. $Y=\bigcup Y_{n}$ where $Y_{m}=Y \cap \mu_{m}$ for $0<m<\omega$. Choose $m \geqq n$ so that $c Y_{m} \geqq \omega_{1}$. Let $X^{\prime}=\left\{x \in X_{n}: f(x) \in Y_{m}\right\}$. Then $f: X^{\prime} \rightarrow Y_{m}, c X^{\prime}=\omega_{1}, f$ is decreasing and $X^{\prime}, Y_{m} \cong \mu_{m}$. Since $\mu_{m} \cong \kappa^{m}$ this is impossible.

To obtain $\mathfrak{A}$, we remove the first element of $\mu$.

Counterexample 4.6. Suppose $\kappa$ is uncountable. There are structures $\mathfrak{A}$ and $\mathfrak{B}$ such that $\mathfrak{A} \equiv \mathfrak{B}(\underline{L})$ but $\mathfrak{A}^{2} \not \equiv \mathfrak{B}^{2}\left(L_{\kappa}^{2}\right)$.

Take $\mathfrak{A}$ to be the order constructed in Lemma 4.5. Take $\mathfrak{B}=$ $\mathfrak{A} \cdot \kappa^{*}$. Here $\kappa^{*}$ is the converse ordering of the ordinal $\kappa$, and $\cdot$ denotes ordinal product. Both $\mathfrak{A}$ and $\mathfrak{B}$ are $\kappa$-dense linear orders without endpoints of power $\kappa$ and so by Theorem $4.2 \mathfrak{A} \equiv \mathfrak{B}(\underline{L})$. $\quad \mathfrak{A}^{2}$ has no antichain of power $\kappa$ so $\mathfrak{A}^{2} \not \#_{\kappa} Q^{2} x y[\neg(x \leqq y) \wedge \neg(y \leqq x)]$. $\mathfrak{B}$ has a suborder of type $\kappa$ and a suborder of type $\kappa^{*}$ so there is $f \subseteq|\mathfrak{B}|^{2}$ so that $f$ is strictly decreasing and card $f \geqq \kappa$. By Lemma 4.4 this means

$$
\mathfrak{B}^{2} \vDash{ }_{\kappa} Q^{2} x y[\neg(x \leqq y) \wedge \neg(y \leqq x)] \text {. }
$$

For the case $\kappa=\omega$ we must construct a different counterexample. In the remainder of this section we will be working in the $\omega$-interpretation; + and $\cdot$ will denote ordinal addition and multiplication and $\lambda$ and $\mu$ will be arbitrary order types. As in Slomson [16], we say that player II has a nice winning strategy in $G_{m}^{n}(\lambda, \mu)$ if player II has a winning strategy in $G_{m}^{n}(\lambda, \mu)$ following which he picks the first element from $\lambda$ or $\mu$ if and only if player $I$ has on the same move just picked the first element from the other order type.

LEMMA 4.7. (i) If player II has a winning strategy in $G_{m}^{n}(\omega+\lambda$, $\omega+\mu)$ then player II has a nice winning strategy in $G_{m}^{n}(\omega+\lambda$, $\omega+\mu)$.

(ii) If player II has a nice winning strategy in $G_{m}^{n}(\lambda, u)$ then player II has a winning strategy in $G_{m+1}^{n}(\omega \cdot \lambda, \omega \cdot \mu)$.

(ii) is an analogue of Lemma 5.2 in [16]. Slomson proves the result in the uncountable interpretation and with $n=1$. His proof goes through without changes in the $\omega$-interpretation with $n=1$. Only minor modifications are needed to deal with the Malitz quantifiers $(n>1)$. (i) is clear because $\omega+\lambda \cong 1+\omega+\lambda$ and $\omega+\mu \cong 1+\omega+\mu$.

LEMMA 4.8 . $\omega^{\omega}(1+\lambda) \equiv \omega^{\omega}(1+\mu)\left(L_{\omega}^{<\omega}\right)$. 
Proof. Let $\mathfrak{A}$ denote $\omega^{\omega}(1+\lambda)$ and $\mathfrak{B}$ denote $\omega^{\omega}(1+\mu)$. Clearly player II has a winning strategy in $G_{1}^{n}(\mathscr{A}, \mathfrak{B})$. So by Lemma 4.7(i) player II has a nice winning strategy in $G_{1}^{n}(\mathfrak{A}, \mathfrak{B})$. Applying 4.7(ii) player II has a winning strategy in $G_{2}^{n}(\omega \cdot \mathfrak{A}, \omega \cdot \mathfrak{B})$. But $\omega \cdot \mathfrak{A}=$ $\omega \cdot\left(\omega^{\omega}(1+\lambda)\right)=\left(\omega \cdot \omega^{\omega}\right)(1+\lambda) \cong \omega^{\omega}(1+\lambda)=\mathfrak{A}$ and similarly $\omega \cdot \mathfrak{B} \cong \mathfrak{B}$. So player II has a winning strategy in $G_{2}^{n}(\mathfrak{A}, \mathfrak{B})$. Repeated applications of Lemma 4.7 yield that for all $m$, player II has a winning strategy in $G_{m}^{n}(\mathfrak{A}, \mathfrak{B})$. Since $n$ is arbitrary, $\mathfrak{A} \equiv \mathfrak{B}\left(L_{\omega}^{<\omega}\right)$.

Counterexample 4.9. There are structures $\mathfrak{A}$ and $\mathfrak{B}$ such that $\mathfrak{A} \equiv \mathfrak{B}\left(L_{\omega}^{<\omega}\right)$ but $\mathfrak{A}^{2} \not \equiv \mathfrak{B}^{2}\left(L_{\omega}^{2}\right)$.

Take $\mathfrak{A}=\left\langle\omega^{\omega}\right.$, $\leqq$ and $\mathfrak{B}=\left\langle\omega^{\omega}\left(1+\omega^{*}\right), \leqq\right\rangle$. By Lemma 4.8 $\mathfrak{A} \equiv \mathfrak{B}\left(L_{\omega}^{<\omega}\right)$. Since $\mathfrak{B}$ has a suborder of type $\omega$ and a suborder of type $\omega^{*}$ there is $f \subseteq|\mathfrak{B}|^{2}$ such that card $f=\omega$ and is $f$ strictly decreasing. Since $\mathfrak{A}$ is an ordinal there is no such $f \subseteq|\mathfrak{A}|^{2}$. By Lemma 4.4 this means that

$$
\begin{aligned}
& \mathfrak{B}^{2} \vDash Q^{2} x y[\neg(x \leqq y) \wedge-(y \leqq x)] \text { and } \\
& \mathfrak{Y}^{2} \not \models Q^{2} x y[-(x \leqq y) \wedge-(y \leqq x)]
\end{aligned}
$$

Since $\mathfrak{A}=\left\langle\omega^{\omega}, \leqq\right\rangle$ is well ordered and $\mathfrak{B}=\left\langle\omega^{\omega}\left(1+\omega^{*}\right), \leqq\right\rangle$ is not well ordered and since $\mathfrak{A} \equiv \mathfrak{B}\left(L_{\omega}^{<\omega}\right)$ we have:

THEOREM 4.10. The notion that a binary relation is a well order is not expressible in $L_{\omega}^{<\omega}$.

5. Craig's theorem. The Counterxamples 4.6 and 4.8 also provide a proof that Craig's theorem fails in these languages. The essential observation is that if we add pairing functions to a structure $\mathfrak{A}$ then the theory of $\mathfrak{A}^{2}$ reduces to the theory of $\mathfrak{A}$ with the pairing functions.

Let $\leqq$ be a binary relation symbol, $P$ and $R$ binary function symbols, and let $\pi_{1}, \pi_{2}, \rho_{1}$, and $\rho_{2}$ be unary function symbols. Let $\phi$ express that $\leqq$ is a linear order, let $\delta$ say that $P, \pi_{1}$, and $\pi_{2}$ are pairing and coordinate functions,

$$
\delta=\forall x \forall y\left[\pi_{1}(P(x, y))=x \wedge \pi_{2}(P(x, y))=y \wedge P\left(\pi_{1}(x), \pi_{2}(x)\right)=x\right]
$$

and let $\gamma$ say that $R, \rho_{1}$ and $\rho_{2}$ are also pairing and coordinate functions. Let

$$
\begin{aligned}
& \sigma_{\delta}=\mho^{2} x y\left[\pi_{1}(x) \leqq \pi_{1}(y) \wedge \pi_{2}(x) \leqq \pi_{2}(y)\right] \text { and } \\
& \sigma_{\gamma}=\vartheta^{2} x y\left[\rho_{1}(x) \leqq \rho_{1}(y) \wedge \rho_{2}(x) \leqq \rho_{2}(y)\right] .
\end{aligned}
$$

Now suppose $\mathbb{C}$ is of type $\leqq, P, \pi_{1}, \pi_{2}, R, \rho_{1}, \rho_{2}$. Some easy facts to check are: 
if $\sqrt{ } \vDash \delta$ then $P^{\mathbb{\complement}}$ maps $|\mathfrak{F}|^{2}$ one-to-one and onto $|\sqrt{ }|$ if $\sqrt{\mathfrak{F}} \vDash \gamma$ then $R^{\mathfrak{\varsigma}}$ maps $|\mathfrak{F}|^{2}$ one-to-one and onto $|\mathfrak{F}|$

if $\sqrt{ } \vDash \rho \wedge \delta$ then $\mathbb{C} \vDash{ }_{\kappa} \sigma_{\delta}$ if and only if $\mathbb{C}^{2}$ has no $\kappa$-powered antichain.

Similarly if $\mathfrak{E} \vDash \varphi \wedge \gamma$ then $\mathbb{E} \vDash{ }_{\kappa} \sigma_{\gamma}$ if and only if $\mathbb{C}^{2}$ has no $\kappa$-powered antichain.

Using these facts we see that

$$
\begin{aligned}
& \vDash\left(\varphi \wedge \delta \wedge \sigma_{\delta}\right) \longrightarrow\left(\gamma \longrightarrow \sigma_{\gamma}\right) . \\
& \varphi \wedge \delta \wedge \sigma_{\delta} \text { is of type } \leqq, P, \pi_{1} \text { and } \pi_{2} \text { and } \\
& \gamma \longrightarrow \sigma_{\gamma} \text { is of type } \leqq, R, \rho_{1}, \text { and } \rho_{2} .
\end{aligned}
$$

We claim that there is no Craig interpolant, $\bar{\sigma}$ in $L_{\kappa}^{<\omega}$ and of type $\leqq$ such that $\vDash\left(\varphi \wedge \delta \wedge \sigma_{\delta}\right) \rightarrow \bar{\sigma}$ and $\vDash \bar{\sigma} \rightarrow\left(\gamma \rightarrow \sigma_{\gamma}\right)$.

Suppose such $\bar{\sigma}$ did exist. We will get a contradiction. If $\kappa=\omega$ let $\mathfrak{A}$ and $\mathfrak{B}$ be as in in Counterexample 4.9, if $\kappa>\omega$ let $\mathfrak{A}$ and $\mathfrak{B}$ be as in Counterexample 4.6. $\mathfrak{A}$ and $\mathfrak{B}$ are both infinite and of type $\leqq$. Since $\mathfrak{A}$ is infinite we can define $P, \pi_{1}$, and $\pi_{2}$ such that $\left\langle|\mathfrak{A}|, \leqq, P, \pi_{1}, \pi_{2}\right\rangle \vDash \delta$. Since $\mathfrak{A}$ is a linear order and $\mathfrak{A}^{2}$ has no $\kappa$ powered antichain we have $\left\langle|\mathfrak{A}|, \leqq, P, \pi_{1}, \pi_{2}\right\rangle \vDash \varphi \wedge \delta \wedge \sigma_{\dot{\delta}}$. So $\left\langle|\mathfrak{A}|, \leqq, P, \pi_{1}, \pi_{2}\right\rangle \vDash \bar{\sigma}$, but $\bar{\sigma}$ is of type $\leqq$ so $\mathfrak{A} \vDash \bar{\sigma}$. $\mathfrak{A} \equiv \mathfrak{B}\left(L_{\kappa}^{<\omega}\right)$ so $\mathfrak{B} \vDash \bar{\sigma}$. Also $\mathfrak{B} \vDash \varnothing$. Since $|\mathfrak{B}|$ is infinite we can define $R, \rho_{1}$, and $\rho_{2}$ such that $\left\langle|\mathfrak{B}|, \leqq, R, \rho_{1}, \rho_{2}\right\rangle \vDash \gamma$. But $\vDash \bar{\sigma} \rightarrow\left(\gamma \rightarrow \sigma_{\gamma}\right)$ so $\langle|\mathfrak{B}|, \leqq$, $\left.R, \rho_{1}, \rho_{2}\right\rangle \vDash \varphi \wedge \gamma \wedge \sigma_{\gamma}$. This means $\mathfrak{B}^{2}$ has no $\kappa$-powered antichain, which contradicts its construction. We have just proved

THEOREM 5.1. Craig's theorem fails in $L_{\kappa}^{<\omega}$ in that there is a valid implication of $L_{\kappa}^{2}$ which has no interpolant in $L_{\kappa}^{<\omega}$. (No assumption $\kappa$.)

This result may be contrasted with the unpublished result of Stavi who showed that if $\kappa$ is uncountable and regular then there is a valid implication of $L_{\kappa}^{1}$ which has no interpolant in $L_{\kappa}^{<\omega}$.

Actually, if we assume $\kappa>\omega$ then we know $\mathfrak{A} \equiv \mathfrak{B}(\underline{L})$. This means we can then strengthen the result to say that there is a valid implication of $L_{k}^{2}$ with no interpolant in $\underline{L}$.

As was pointed out in [2] a more general result which implies Theorem 5.1 is that which says that if $L$ is any language in which theory of the square of a structure is interpretable into the theory of the structure with pairing functions added, if in $L$ one can express the notion of pairing functions, and if there are two infinite structures whose squares do not preserve their $L$ equivalence, then Craig's theorem must fail in $L$. 


\section{REFERENCES}

1. L. Badger, An Ehrenfeucht game for $L^{<\omega}$, A. M. S. Notices, 21, No. 1 (1974), A19, Abstract 711-02-6.

2. - The Malitz Quantifier Meets its Ehrenfeucht Game, Ph. D. Thesis, University of Colorado, 1975.

3. W. Brown, Infinitary Languages, Generalized Quantifiers, and Generalized Product Ph. D. Thesis, Dartmouth University, August, 1971.

4. Chang and Keisler, Model Theory, Amsterdam, North-Holland Co., 1973, 550 pp.

5. W. Craig, Linear Reasoning, A new form of the Herbrand-Gentzen theorem, J. Symbolic Logic, 22, No. 3 (1957), 250-268.

6. A. Ehrenfeucht, An application of games to the completeness problems for formalized theories, Fund. Math., 49 (1961), 129-141.

7. Feferman and Vaught, First order properties of products of algebraic systems, Fund. Math., 47 (1959), 57-103.

8. R. Fraïsé, Sur quelques classifications des systèmes de relations, Publ. Sci. Univ. Alger Ser. A, 1 (1954), 35-182.

9. G. Fuhrken, Skolem-type normal forms for first order languages with a generalized quantifier, Fund. Math., 54 (1964), 291-302.

10. - Languages with added quantifier "there exists a least $\boldsymbol{\aleph}_{\alpha}$," The Theory of Models, Proceedings of the 1963 International Symposium at Berkeley, Amsterdam, North-Holland Co., 1965, 121-131.

11. C. Karp, Finite quantifier equivalence, The Theory of Models, Proceedings of the 1963 International Symposium at Berkeley, Amsterdam, North-Holland Co., (1965), 407-412. 12. J. Keisler, Logic with the quantifier "there exist uncountably many," Ann. of Math. Logic, 1 (1970), 1-93.

13. L. Lipner, Some Aspects of Generalized Quantifiers, Ph. D. thesis, University of California, Berkeley, 1970.

14. Magidor and Malitz, Compact extensions of $L(Q)$ (part 1a), to appear.

15. A. Mostowski, On direct products of theories, J. of Symbolic Logic, 17 (1952), 1-31.

16. A. B. Slomson, Generalized quantifiers and well orderings, Arch. Math. Logik. 15 (1972), 57-73.

17. R. Vaught, The completeness of logic with the added quantifier "there are uncountably many", Fund. Math., 54 (1964), 303-304.

18. S. Vinner, A generalization of Ehrenfeucht's game and some applications, Israel J. of Math., 12, No. 3 (1972), 279-298.

19. A. Wojciechowska, Generalized products for $Q_{\alpha}$-languages, Bull. de L'Acad. Polon. des Sci., Série des sci. math., astr. et phys., 17, No. 6 (1969), 337-339.

Received October 27, 1976 and in revised form April 26, 1977. This material appeared in the author's doctoral dissertation which was prepared under the kind guidance of Professor Jerome Malitz. (University of Colorado, 1975). The research was partially supported by N. S. F. grants GP-28070 and GP-19405. In addition to Professor Malitz, the author would like to thank Menachem Magidor and Bill Coulter for many stimulating discussions concerning the model theory of the Malitz quantifiers.

SOUTHERN ILLINOIS UNIVERSITY

Carbondale, IL 62901 


\title{
PACIFIC JOURNAL OF MATHEMATICS
}

\section{EDITORS}

\author{
RICHARD ARENS (Managing Editor) \\ University of California \\ Los Angeles, CA 90024 \\ Charles W. Curtis \\ University of Oregon \\ Eugene, OR 97403 \\ C. C. MOORE \\ University of California \\ Berkeley, CA 94720
}

\section{J. DUGUNDJI}

Department of Mathematics

University of Southern California

Los Angeles, CA 90007

R. FINN and J. MILGRAM

Stanford University

Stanford, CA 94305

\section{ASSOCIATE EDITORS}
E. F. BECKENBACH
B. H. NeumanN
F. WOLF
K. YoSHIDA

\section{SUPPORTING INSTITUTIONS}

UNIVERSITY OF BRITISH COLUMBIA

CALIFORNIA INSTITUTE OF TECHNOLOGY

UNIVERSITY OF CALIFORNIA

MONTANA STATE UNIVERSITY

UNIVERSITY OF NEVADA, RENO

NEW MEXICO STATE UNIVERSITY

OREGON STATE UNIVERSITY

UNIVERSITY OF OREGON

OSAKA UNIVERSITY

\author{
UNIVERSITY OF SOUTHERN CALIFORNIA \\ STANFORD UNIVERSITY \\ UNIVERSITY OF HAWAII \\ UNIVERSITY OF TOKYO \\ UNIVERSITY OF UTAH \\ WASHINGTON STATE UNIVERSITY \\ UNIVERSITY OF WASHINGTON \\ $\stackrel{*}{*} \stackrel{*}{*} \stackrel{*}{*}$ AMERICAN MATHEMATICAL SOCIETY
}

The Supporting Institutions listed above contribute to the cost of publication of this Journal, but they are not owners or publishers and have no responsibility for its content or policies.

Mathematical papers intended for publication in the Pacific Jaurnal of Mathematics should be in typed form or offset-reproduced, (not dittoed), double spaced with large margins. Please do not use built up fractions in the text of your manuscript. You may however, use them in the displayed equations. Underline Greek letters in red, German in green, and script in blue. The first paragraph or two must be capable of being used separately as a synopsis of the entire paper. Items of the bibliography should not be cited there unless absolutely necessary, in which case they must be identified by author and Journal, rather than by item number. Manuscripts, in triplicate, may be sent to any one of the editors. Please classify according to the scheme of Math. Reviews, Index to Vol. 39. All other communications should be addressed to the managing editor, or Elaine Barth, University of California, Los Angeles, California, 90024.

The Pacific Journal of Mathematics expects the author's institution to pay page charges, and reserves the right to delay publication for nonpayment of charges in case of financial emergency.

100 reprints are provided free for each article, only if page charges have been substantially paid. Additional copies may be obtained at cost in multiples of 50 .

The Pacific Journal of Mathematics is issued monthly as of January 1966. Regular subscription rate: $\$ 7200$ a year (6 Vols., 12 issues). Special rate: $\$ 36.00$ a year to individual members of supporting institutions.

Subscriptions, orders for back numbers, and changes of address should be sent to Pacific Journal of Mathematics, 103 Highland Boulevard, Berkeley, California, 94708.

PUBLISHED BY PACIFIC JOURNAL OF MATHEMATICS, A NON-PROFIT CORPORATION

Printed at Kokusai Bunken Insatsusha (International Academic Printing Co., Ltd.). 8-8, 3-chome, Takadanobaba, Shinjuku-ku, Tokyo 160, Japan.

Copyright (C) 1975 by Pacific Journal of Mathematics Manufactured and first issued in Japan 


\section{Pacific Journal of Mathematics}

Vol. 72, No. 2

February, 1977

George E. Andrews, Plane partitions. II. The equivalence of the

Bender-Knuth and MacMahon conjectures ................. 283

Lee Wilson Badger, An Ehrenfeucht game for the multivariable quantifiers

of Malitz and some applications ......................... 293

Wayne C. Bell, A decomposition of additive set functions ............ 305

Bruce Blackadar, Infinite tensor products of $C^{*}$-algebras ............. 313

Arne Brøndsted, The inner aperture of a convex set .............. 335

N. Burgoyne, Finite groups with Chevalley-type components........... 341

Richard Dowell Byrd, Justin Thomas Lloyd and Roberto A. Mena, On the retractability of some one-relator groups .....................

Paul Robert Chernoff, Schrödinger and Dirac operators with singular potentials and hyperbolic equations .................... 361

John J. F. Fournier, Sharpness in Young's inequality for convolution ....... 383

Stanley Phillip Franklin and Barbara V. Smith Thomas, On the metrizability

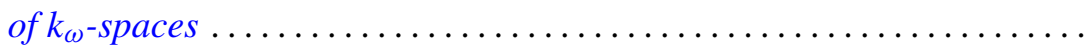

David Andrew Gay, Andrew McDaniel and William Yslas Vélez, Partially normal radical extensions of the rationals .................... 403

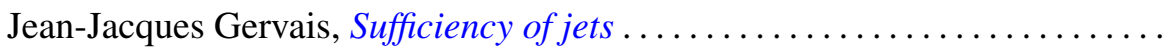

Kenneth R. Goodearl, Completions of regular rings. II . .

Sarah J. Gottlieb, Algebraic automorphisms of algebraic groups with stable maximal tori

Donald Gordon James, Invariant submodules of unimodular Hermitian forms.....

J. Kyle, $W_{\delta}(T)$ is convex.

Ernest A. Michael and Mary Ellen Rudin, A note on Eberlein compacts ...

Ernest A. Michael and Mary Ellen Rudin, Another note on Eberlein compacts ....

Thomas Bourque Muenzenberger and Raymond Earl Smithson, Fixed point theorems for acyclic and dendritic spaces.

Budh Singh Nashier and A. R. Rajwade, Determination of a unique solution of the quadratic partition for primes $p \equiv 1(\bmod 7)$.

Frederick J. Scott, New partial asymptotic stability results for nonlinear ordinary differential equations ....................

Frank Servedio, Affine open orbits, reductive isotropy groups, and dominant gradient morphisms; a theorem of Mikio Sato..........

D. Suryanarayana, On the distribution of some generalized square-full integers.................................. 